\title{
Dialog Mapping: Reflections on an Industrial Strength Case Study
}

\author{
Jeff Conklin
}

CogNexus Institute, and George Mason University, USA

\section{Introduction}

Earlier chapters have introduced the notion of "wicked problems" and the Issue Based Information System (IBIS) framework (Buckingham Shum, Chapter 1; van Bruggen et al, Chapter 2), both of which derive from the work of Horst Rittel. Selvin (Chapter 7) proposes a generic framework for facilitated Computer Supported Argument Visualization (CSAV), and reports on case studies using the IBISbased Compendium approach. Compendium itself is based on a facilitated CSAV approach called Dialog Mapping, the focus of this chapter. We begin by elaborating on the art and process of Dialog Mapping, before reporting on a particular business application, probably the longest-term case study available of CSAV adoption in an organization.

The case study reports on ten years of continuous usage of Dialog Mapping by a group of approximately 50 users in the Environmental Affairs division of Southern California Edison (SCE). More precisely, this group have been users of the QuestMap ${ }^{\mathrm{TM}}$ software system², which is the software system underpinning Dialog Mapping. QuestMap provides some hypertext and groupware features which are quite powerful but are also can be difficult for new users to master. Such features often spell doom for the successful rollout of new collaboration technologies. The case study explores the some of the practical success factors for CSAV adoption as they apply to the case of the adoption and usage of QuestMap and Dialog Mapping at SCE.

\section{IBIS: Issue-Based Information System}

At the heart of the Dialog Mapping approach is the IBIS argumentation system. IBIS is a rhetorical "grammar" which defines the basic elements of all analysis and design dialogs. IBIS consists of three basic elements: Questions, which pose a problem or issue, Ideas, which offer possible solutions or explanations to the Questions, and Arguments, which state evidence, facts, and viewpoints that either support or object to Ideas. Also called "pros and cons", arguments are often linked to evidential documents that back up their claim. IBIS was developed in the early 1970's as a tool to support planning and policy design processes (Kunz and Rittel, 1970).

For example, Figure 1 shows a small IBIS map for a meeting of a school board faced with a budget shortfall. Generally these maps are constructed from left to right; thus the "root issue" in this map is "What should we do about the budget?" There are three possible answers ("ideas") in the map so far, and there are additional questions about the first two ideas. There is an argument against the first idea,

1 This paper appears as a chapter in Visualizing Argumentation: Software Tools for Collaborative and Educational Sense-Making, Kirschner, P.A., Buckingham Shum, S.J., \& Carr, C.S., Eds., Springer, London, 2003. See http://www.visualizingargumentation.info/ for more information.

2 QuestMap is a trademark of Touchstone Consulting, Inc., Washington, DC, USA 
and an argument for the third idea. The links may seem to be backward, but they are not: new nodes are about existing nodes, and so point back to them ${ }^{3}$.

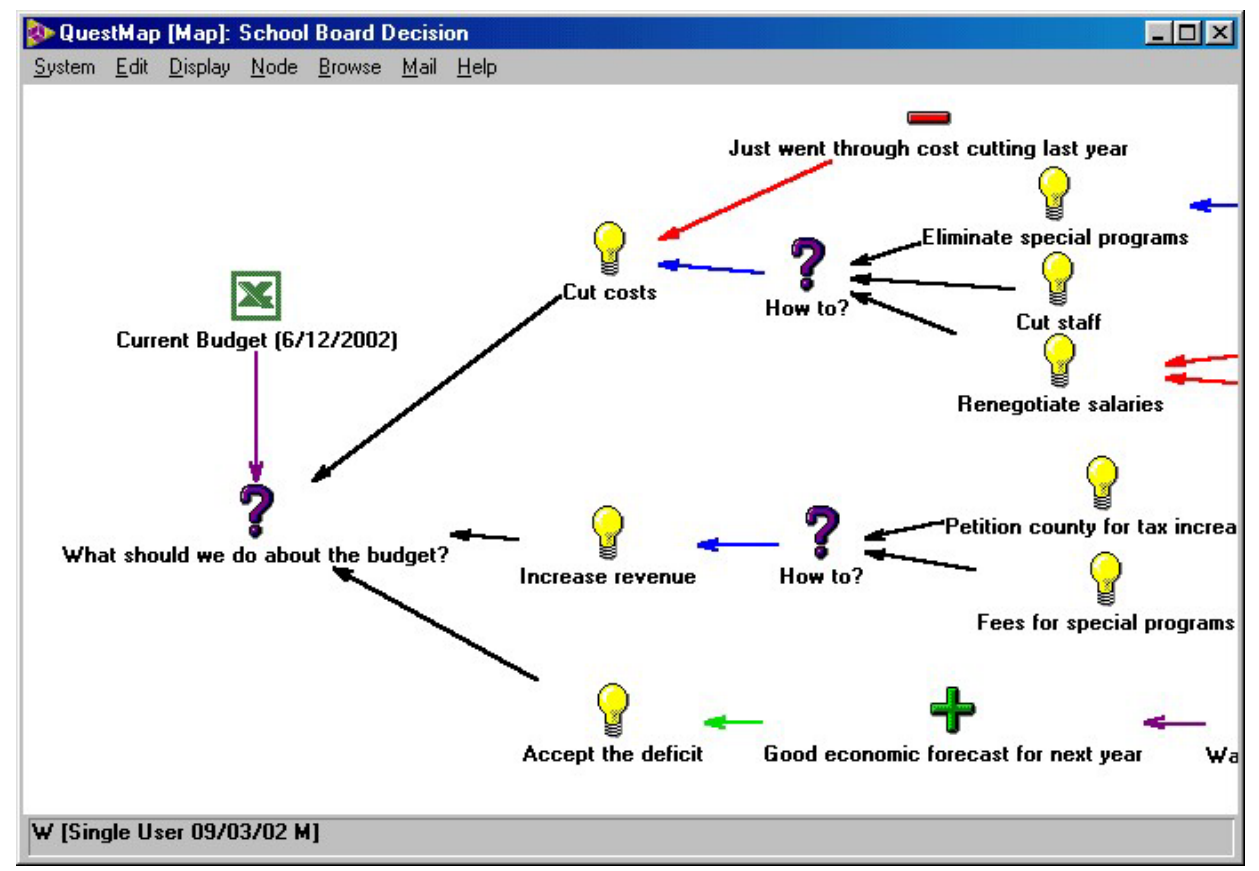

Figure 1: Dialog map segment showing all three IBIS elements. Note the spreadsheet program icon in the upper left which is a hot link to a background source document for the discussion.

The power of IBIS stems from three properties: (i) it maps complex thinking into structured analytic maps; (ii) it is based on asking the right questions; and (iii) it is simple and intuitive enough to be learned and used with relatively low cognitive overhead (making it a good cognitive encoding formalism). Let's consider each of these claims in more detail.

First, IBIS captures complex thinking in structured analytic maps (i.e., diagrams). There is no limit to the size or richness of IBIS maps. A variety of linking and navigation mechanisms provide users with several ways to organize large amounts of information. One of the most basic is that a map can contain other maps (represented as map icons), so that information can be logically chunked. Often, the signal for a new chunk, a new map, is a major new question. For example, in Figure 1, the exploration of how to cut costs might lead to a larger and more detailed argument structure which would be better moved into its own map. This new "sub-map" would be hyperlinked to the top-level map.

Moreover, unlike most diagramming and mapping techniques ${ }^{5}$, IBIS has a grammar, imposing a formal structure (albeit a simple one) on IBIS maps. In English, you cannot say "John ball hit the." In IBIS, maps never start with an Argument node, for example, nor are Arguments allowed to link to Questions. The IBIS grammar imposes a discipline on IBIS maps, with two complementary consequences: it is harder to learn to use IBIS than to use free-form techniques ${ }^{6}$, and IBIS maps are

3 Thus the direction of the arrow is connected to the semantics of the link. For example, the idea "Cut costs" is about - "responds to" - the question "What should we do about the budget?" The impression that the link points backwards generally fades quickly.

4 Active use of any formalism will involve some cognitive effort. Typically one can become proficient in IBIS after a 2-day course and some practice, with the emphasis on practice: you become fluent in a new language only by conversing in it.

5 For example, some consultants use the "mind mapping" approach and a tool that supports it, "MindManager" (for detail see www.mindjet.com/)

6 "Free form" is equivalent to having a trivial or very simple grammar, e.g. circles and arrows. 
more rigorous, more patterned, and more reproducible. For the analysis of wicked situations, the additional structure and rigor of IBIS creates an important baseline of order and reproducibility.

Second, IBIS invokes a discipline of finding the right questions. Recall that "IBIS" stands for "Issue Based Information System". Issues, stated as questions, are the heart of this method. One benefit of asking questions in a collaborative situation is that it can help to break up the "answer reflex," in which participants exchange answers without ever agreeing on the question. Also, the IBIS grammar allows a Question to be about any other IBIS element, so it is through asking new questions that IBIS maps grow. Finally, there are a small number of types of questions in IBIS (approximately five) - each type calls for different kinds of responses, and these types connect together in higher-level patterns, or templates. These patterns provide additional order and reproducibility in IBIS maps.

For example, there are Factual questions, such as "What is the expected budget shortfall?" There are Future questions, such as "What will the economy be like next year?" There are Meaning questions, such as "What are "special programs'?" Each type of question has certain kinds of answers, and they fit together in regular patterns of reasoning.

The third important property of the IBIS grammar is that it is simple and intuitive. Numerous studies have shown that the potential advantages of rhetorical structuring techniques like IBIS are often offset by the increased "cognitive overhead" of applying them when engaged in a substantive task (Buckingham Shum and Hammond, 1994). In part, this overhead imposes a constraint on the expressive power of any formalism that might be adopted in the analysis process: candidate notations must be simple and intuitive enough that the "cognitive cost" of using them is very low. Years of practical experience have shown that more sophisticated and expressively powerful notations than IBIS are much more difficult to apply in a transparent way.

There is another way to meet the challenge of "cognitive overhead," and that is to recognize that lowering the cognitive cost of use is in part a matter of fluency. Although English is generally recognized as one of the more difficult languages to learn in the world, fluent speakers are unaware of the "cognitive overhead" of English when they are engaged in discussion. English is transparent to both speakers and listeners who are fluent. Similarly, as with a language or a musical instrument, practice and fluency render IBIS both transparent and powerful.

As simple as IBIS is, it can be broken down even further to increase its simplicity for those in the learning phase. One part of IBIS is Questions and Ideas/Answers, the basic constituents of dialog dating back to Aristotle. Virtually anyone can listen to a debate and quickly discern what the question is (or questions are), and what the positions or possible answers are. Moreover, the majority of comments in an analytic discussion are questions and answers, so you can map much of these interactions using only these two IBIS elements.

More challenging for IBIS students is the proper use of Arguments, the pros and cons for the various Ideas. Of course, the concept of Arguments is simple and intuitive. Most people are quite used to thinking in terms of tradeoffs, benefits and disadvantages, strengths and weaknesses, opportunities and a threats, and so on. These are the basic terms of critical analysis. However, there are subtle pitfalls for the unpracticed. For example, some arguments clearly object to an idea, e.g. "Management will reject this." But sometimes an argument supports an idea, but because it contains "negative words" it gets erroneously linked as an objection, e.g. "Rejection is unlikely." In applying Arguments clearly and correctly a background in logic can come in handy! In any case, it turns out that only about $10 \%$ of the nodes in open-ended and exploratory discussions are Argument nodes, and even less in more structured analyses, so, again, these are the least important of the IBIS elements (Selvin and Sierhuis, 1999).

In summary, distinguishing the three basic elements of IBIS - Questions, Ideas, and Arguments can become, with practice, very natural. Indeed, many people report that, having learned IBIS, they find it very frustrating to listen to discussions in which the participants are not making these basic rhetorical distinctions! (We explore some ways of dealing with training costs and the learning curve later) Moreover, participants in meetings in which IBIS mapping is done on the fly, either with markers on a white board or with software on a computer projector, find the notation quite natural and obvious. No explanation is necessary, and any sense of mystery about what is going on generally vanishes quickly - assuming, of course, some level of fluency in the person doing the mapping!

One final note on IBIS: in addition to how simple and natural this notation is, it is also important to convey the power of IBIS as a mapping notation for complex analyses. It is easiest to see this power simply by reflecting on what happens without such a notation. Different players have different ideas 
about what the issue is, especially in a wicked situation. Each player addresses their comments to their version of the issue, but it is often unclear how many versions of the issue there are, or what they are. Making the issues explicit in an IBIS map, as questions, adds a tremendous clarity to the discussion, as does being clear about which of those issues a given comment is addressing. Wicked problems often have dozens of interrelated issues involved, but human short term memory is limited, so unless one is extremely familiar with the all of the information related to all of the issues, the unaided exploration of these issues is confusing and error-prone. The power of IBIS as a notation is that it organizes all of the issues, positions, information, and assumptions so that all participants have the issue map as a point of reference, and they can refer to it instead of trying to keep it all in their head. Thus IBIS contributes to Dialog Mapping being a "cognitive aid" because it augments human cognition at one of its weakest points: the limits of short term memory.

\subsection{Artful Questions}

Much of the power of IBIS derives from its artful application, and nowhere is that art more evident than in the creation of questions. Artful questions must be simple, not compound. ("How big is the budget shortfall, and what should we do about it?" is a compound question - in IBIS it should be broken into its two component questions.) Artful questions do not try to "sneak" assumptions into their statement (e.g. "Given that we are already understaffed, what should we do?"). And artful questions are open, not closed. ("What should we do?" is open, "Should we cut costs to meet the budget?" is closed.)

A closed question is one for which the answer is "yes" or "no", or which lists the possible answers in the question (e.g. "Should we cut costs to meet the budget?"). Closed questions contain the answer and simply ask for verification. Years of experience have shown that IBIS discussions that start with open questions are more creative and rigorous. Closed questions seem to "stack the deck" and close the mind to new possibilities.

And yet, closed questions are very common in everyday speech. Many meetings have closed questions as their agenda, and occasionally facilitators are called on by their client to help a group address closed questions.

Fortunately, IBIS makes it easy to "open" the question up. Suppose the starting question is "Should we cut costs?" To open the question, simply locate the answer buried inside the question (in this case, "cut costs") and restate the question with the answer as an Idea: "What should we do?", "Cut costs". All of the analysis about cutting costs can then be attached to the idea "Cut costs" (see Figure 2).

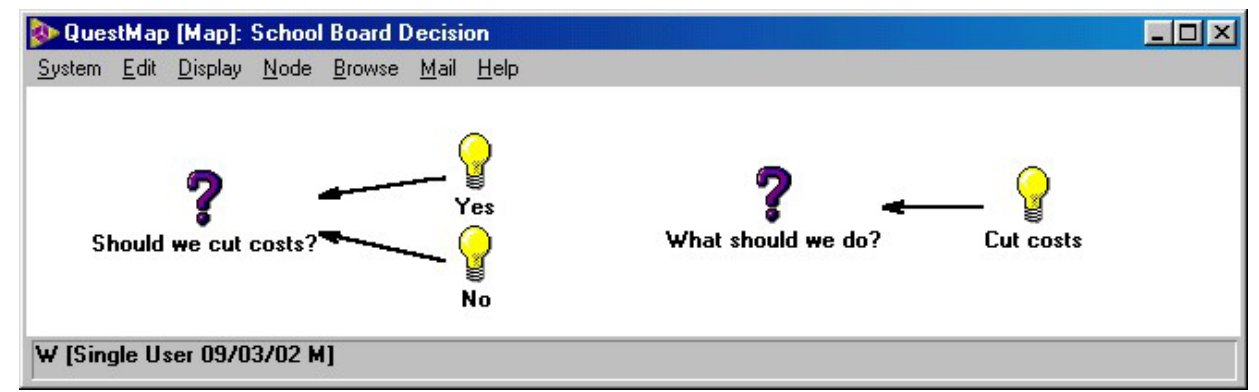

Figure 2: Opening up a question in IBIS. The forms are logically equivalent: the same arguments, issues, and evidence that apply to "Yes" on the left apply to "Cut costs" on the right.

Opening up questions is one simple example of how IBIS can support creative and rigorous analysis of a situation ... without putting the project group in a linear process or methodological straightjacket.

\section{Dialog Mapping}

Dialog Mapping is a facilitation technique in which the Facilitator captures the group's discussion in real time in an IBIS map. Dialog Mapping can be done on a piece of paper or a flipchart pad, on a 
whiteboard, or using software such as QuestMap7. Dialog Mapping is a major aspect of the Compendium technique described by Selvin in Chapter 7; all of this chapter's discussion about Dialog Mapping applies to Compendium as well, but the focus here is on Dialog Mapping.

The role of the Facilitator has two component roles: one role includes engaging with the group in traditional facilitative ways (i.e. watching the clock and keeping the group on task), and the other role, also known as "technographer," focuses on listening and typing at the computer. Although one person can perform both roles, it is often preferable if two people work together, one as Facilitator and one as Technographer. In this paper we will refer simply to "the Facilitator."

In Dialog Mapping the group actively and collaboratively engages with the creation of the IBIS map. Central to Dialog Mapping is the notion of a "shared display", a projected computer display of hypertext software showing a map of the knowledge and reasoning of the group, unfolding in real time.

Dialog Mapping works because of the skill of the Facilitator. The Facilitator is sometimes referred to as a "chauffeur," because (unlike traditional facilitation) the Facilitator's job is to serve as an unobtrusive link between the group's conversation and the shared map. If group members are only speaking to each other and not looking at the shared map, then the Facilitator is not doing his or her job. Skillful Facilitation means listening to the comments of the group and capturing them in the map in a way that clarifies meaning and adds value to the conversation. The group learns to look at the map because, when it doesn't, it gets lost or goes in circles. The Facilitator is in charge of listening, and thus assures that there is a high quality of communication present in the session. In essence, the process of Dialog Mapping focuses a group on constructing a collectively owned model of their problem. With this visual model evolving as they speak, discussions revolve around agreeing on the model, which may of course include agreeing on points of disagreement.

Of course, sometimes the group doesn't pay much attention to the map, and occasionally the group wants the mapping to go faster or to go in a different direction. As with any group intervention, there are dozens of factors and parameters that contribute to the success or failure of a given Dialog Mapping session. One of the most important skills a Facilitator must learn is keeping the group focused on the map and maintaining the group's ownership of the map. Another critical skill is the ability to listen and type at the same times.

Because Dialog Mapping is about the transparent crafting of a high quality map of the group's thinking, sometimes a Facilitator just listens, types, and edits the map. At other times more intervention is called for, including process guidance such as suggesting topics or calling for a break. The Facilitator must sense the needs of the group and make judgments about what kinds of process intervention and guidance, if any, are appropriate. Dialog Mapping does not work in every kind of meeting or with every kind of group. Sometimes the map ends up being nothing more than fancy meeting minutes.

To summarise, a common problem in collective work is sorting out what level of detail and abstraction the group needs to be focused on at any moment. For example, one of the most challenging collaborative tasks - in any field - is collaborative writing. In part this is because participants are working to create and organize the ideas (content) of the document at the same time that they are working on the expression of those ideas (style, wording, grammar, spelling, etc.). This often leads to unproductive disagreements (e.g. the "happy/glad" debate: whether to use "happy" or "glad" in a sentence) as participants stumble between content and style. Moreover, this kind of disagreement can mask a deeper lack of shared understanding about the issues and concepts, resulting in endless and irresolvable disputes over wording.

Dialog Mapping addresses this problem by providing a "pure content" framework - a semistructured model - for the problem at hand. Participants can stay focused on the content level because it is starkly and graphically represented in the shared hypertext display. Moreover, disagreements over content are not mere verbal jousting matches - the Facilitator works with the group to bring the participants' best thinking to mapping out the opposing options and their respective arguments, and perhaps even introducing new options. While this emotional distancing does not make the debate

7 Dialog Mapping is described here in the face-to-face meeting context; the process is similar, but with some differences, in facilitating a virtual meeting.

8 There are many very good traditional facilitators who do not have great typing skills. It is important to bear in mind that Dialog Mapping Facilitation requires an unusual mix of skills, or two people: one to facilitate in the traditional sense and one to be the "technographer." 
disappear, it removes its fangs, and allows clearer heads to negotiate new meanings and formulate new options. In many cases, a clear "winner" emerges simply through the process of open dialog, and even when this doesn't happen, the Dialog Mapping model presents the options and tradeoffs clearly to the decision making authority (i.e. the chair of the meeting).

\section{Case Study: A Decade's Deployment in a Public Utility}

\subsection{Background}

In the Spring of 1992, Michael Begeman and I met with then Executive Vice President David Fogarty and Dr. Michael Hertel, the Director of the Environmental Affairs Division at Southern California Edison (SCE). Using screenshots of a research prototype, ${ }^{9}$ we presented the plans for our new software product, CM/1 (now called QuestMap), explaining that it could be used to capture operational decisions and the rationale behind them, creating an "organizational memory." Early in the presentation, Hertel interrupted our presentation. "If you are telling me what I think you are telling me, " he said slowly, "I am very excited, because I have been looking for something like this for a long time."

That was a fateful moment for the new company, Corporate Memory Systems, Inc., because it was the beginning of the first installation (the "beta site") of our new IBIS-based CSAV system, and our first software sale. Starting with a 20-user license, SCE expanded over the years to its present installation of about 50 users, some of whom have been users of the system for the decade since.

From the tone of that meeting, and the apparent ease with which we had found this customer, the founders of Corporate Memory Systems, Inc. (CMSI) assumed that we had "struck it rich" by tapping into a large market of organizations who would pay well for tools that helped them to capture their organizational learning and memory. Alas, the market was not as ready as we had hoped. Five years later, in 1996, CMSI went out of business due to a lack of sales.

This case study attempts to capture some of the highlights of the SCE story, and to understand what factors have allowed the staff of that organization to continue for ten years to apply a structured argumentation method to the routine issues of environmental planning, regulatory compliance, and policy design.

\subsection{Factors Impacting Success}

In the workshop where this work was first presented ${ }^{10}$, four factors that impact the success or failure of a CSAV system were listed in the call for papers:

- Domain and argumentation knowledge

- Training in tools

- User interface design

- Motivation to use the approach

In a business application of a CSAV system like SCE's, we also want to consider:

- For what organizational purposes was the system used?

- What business problems did it address or solve?

- How was the system used in practice?

- For the people who used the system, why did they continue to use it?

- For those who didn't, why not?

9 The gIBIS (graphical-IBIS) system was developed during the late 1980's in the Software Technology Program at MCC in Austin, Texas, and was the subject of numerous publications in the hypertext and groupware literature (e.g. Conklin \& Begeman, 1988, 1989). See also Buckingham Shum's historical review in Chapter 1.

10 Computer-Supported Collaborative Argumentation for Learning Communities, 11-12 Dec., 1999, Stanford University, CA < www.kmi.open.ac.uk/sbs/csca/csc199> 
- What if any technology features were especially useful?

- What features got in the way?

- Were there features that made believers of advanced users but were barriers to new users?

Moreover, there is the challenge of determining if indeed a CSAV system is a success. Sometimes in business situations one of the hardest issues is "What are the criteria for success?" I now discuss how the above factors had a bearing on SCE's use of QuestMap and Dialog Mapping.

\subsection{Domain and Argumentation Knowledge}

Staff members of the Environmental Affairs Division (EAD) are professionals with high levels of education and training in the areas of environmental and policy science. The EAD has 3 subgroups: a research group that focuses on environmental impacts, a services group focused on regulatory compliance and engineering assistance to facility operators, and an legislative policy group that designs policy and serves as a liaison to state and national environmental agencies. In addition, the EAD makes fairly heavy use of SCE's legal services group.

None of the EAD staff had any previous experience with an argumentation method such as IBIS. The initial training session for the user group was a 3-day course in both IBIS and QuestMap, with emphasis on the software. However, only person (Hertel) used the system following this training. To address this problem, additional training events focused on developing more proficiency in using IBIS as a "language" for thinking (Buckingham Shum and Hammond, 1994), and in coaching smaller work groups that were focused on a specific project and could commit to using the IBIS/QuestMap approach over a specific period of time.

Over the years, additional new users were trained in 2- or 3-day courses and there were refresher courses and advanced workshops for more seasoned users of the system. These courses attempted to cover three areas: the fundamentals of using IBIS as an argumentation structure, the fundamentals of using the QuestMap system, and practice with using IBIS and QuestMap. The issue of training is returned to later (section 4.7, Training Revisited), since it proved so crucial.

\subsection{Training in Tools}

Teaching QuestMap to new users is an interesting challenge. At the level of fundamentals, the software is easy to learn: "The software was incredibly easy to use. I think anybody who is facile with Windows can learn the scheme and use it effectively within an hour or less" (Seybold, 1993). On the other hand, QuestMap has some very sophisticated hypertext features, and the notion of a "virtual shared space", or Common Information Space (CIS - Bannon and Bødker, 1997), was and is only intuitive for more technical users. For example, if a user made a copy of a Question node, pasted it into another view, and edited it, the original node also changed: QuestMap's "Copy" operation was a "shallow" copy, creating a reference link to an underlying database object. Users who did not understand this shared object aspect continued to operate in a private desktop mode, sometimes wreaking havoc with other occupants of the CIS from the changes they made.

Some non-technical users who have been using QuestMap for years still do not understand how and when to use these sophisticated hypertext capabilities. Whether this is because of poor training or a poor user interface, there is not much evidence for successful training in using the "power tools" of the QuestMap system. In more recent years, newcomers to the EAD have picked up using the system more easily, perhaps because they are coming into an environment where it is an established way of working and there are many people who can answer technical questions. 


\subsection{User Interface Design}

QuestMap was originally designed as a groupware CSAV tool for asynchronous collaboration, with additional provisions for "Reference" nodes and "Note" nodes that made it also an attractive CIS tool ${ }^{11}$. Creating and operating on IBIS structures is very easy in QuestMap.12

At SCE, two distinct applications of QuestMap have emerged in the past several years, exploiting two distinct sets of system features. The first is as a File Tracking System, in which users create maps dominated by sub-maps, Note and Reference nodes. The other application is as a Dialog Mapping tool during face-to-face meetings, where one person (often Hertel) "drives" QuestMap, capturing comments in the IBIS notation. ${ }^{13}$ Hertel reports that this technique "works really well," because everyone is paying attention to the "shared display."

Thus at this site (as at others) there appear to be two primary applications of QuestMap, exploiting two aspects of its interface and functionality: as a CIS system for asynchronous information management and sharing, and as a meeting facilitation tool for face-to-face meetings. The former application makes light use of IBIS argumentation, the latter one makes very heavy use of it. The question is, could these be implemented as two separate applications, or is the convergence of synchronous and asynchronous use an essential aspect of its utility? This question is explored further below (section 4.10, Synchronous versus Asynchronous CSAV).

\subsection{Motivation to Use}

The critical factor in the sustained use of QuestMap in EAD is clearly the commitment of Dr. Hertel, the Director. He has provided substantial levels of training, encouraged and occasionally even coerced his staff to use the system, and has been a role model by using QuestMap heavily, not only for visible Division functions but also as a personal note-taking tool when he is on the road at conferences.

Not everyone in the EA Division was successfully motivated to use IBIS and QuestMap. Some people reacted strongly to the early emphasis on having "virtual meetings" within QuestMap instead of face-to-face meetings. Some viewed it as just another "passing fad" of management. Some felt that IBIS was restrictive to the natural flow of conversations, and that using it for meeting facilitation slowed meetings down too much. A deeper study of EAD is planned to explore this resistance.

The other critical motivation to using CSAV has been having a clear business need for either the design rigour provided by embracing the CSAV discipline or the "audit trail" of the decision rationale, or both. In cases where there was a small, focused work group, including someone acting as the "view keeper", ${ }^{14}$ there were many successful extended uses of QuestMap. These groups found that "it really does make it easy to put everything in one place and remember the thinking patterns that you went through to get to certain conclusions, such as why you decided NOT to take a path, which is almost always as important as why you decided TO take one ..." (Seybold, 1993).

\subsection{Training Revisited}

Perhaps the most significant factor in the later success (and early failure) of Dialog Mapping at SCE has been training. It became apparent that teaching QuestMap and IBIS in the same course was trying

11 QuestMap won the award for "Best of Show" in the Group Information Sharing category at the first International Groupware Conference in San Jose in 1992, beating out such heavy weights as Lotus Notes.

12 For some users the mouse is overloaded: creating links and scrolling are both "Right Drag" operations, which is an unusual gesture in the Windows world.

13 Using QuestMap in face-to-face meetings was a client (Hertel) innovation, which CMSI initially resisted (because we had developed QuestMap as an asynchronous groupware system). Fortunately, Hertel insisted, and in fact used large group policy design sessions as a way to introduce QuestMap and CSAV to his organization (Seybold, 1993).

14 Most groups that collaboratively construct IBIS maps in QuestMap end up with very large and poorly structured maps. As a result, CMSI recommended that one participant in a project function as a moderator and gatekeeper for the map, to keep the map orderly and focused and the IBIS structures clean and well-formed. This role was dubbed the "View Keeper". 
to tackle too much at once. Feedback from participants, some weeks after the courses (at this and other QuestMap sites), revealed that many people had not acquired any fluency in using IBIS, while others had only the weakest grasp of the groupware capabilities of QuestMap. In more recent years we have run separate courses for the argumentation and software components (starting usually with the argumentation part), and this has resulted in much better sustained learning.

Indeed, for the past few years, I have given up on training people to use QuestMap altogether, focusing instead on raising IBIS "literacy" in client organizations. In the most recent version of this approach, Dialog Mapping, individuals and groups take the 2-day training course simply to learn a new method for "group problem solving and decision making" that focuses on creating "shared understanding" among the participants. The QuestMap software is provided at no cost, and with only one hour of software-specific training, as a part of the course.

Advanced practice sessions in early 1999 that focused primarily on IBIS and Dialog Mapping had a strong user uptake, particularly among new staff members who "did not have the baggage of the past" (Jim Young, EAD staff member, personal communication). In these sessions, a group of managers who had been trained in both IBIS and QuestMap observed while I facilitated the group in a 2-day strategic planning session (on a real problem of extremely high relevance to the group) using QuestMap. Several people reported learning more than they expected from watching me use the tools.

\subsection{What is "Success"?}

What would constitute "successful adoption" of a CSAV system in an industrial strength setting like SCE? What percentage of users would need to achieve what level of proficiency and sustained usage? What business outcomes would constitute success? How long would the usage of the CSAV system need to be sustained to say that it had "stuck"?

My assessment is that at SCE we have a qualified and perhaps "brittle" success of industrialstrength CSAV. It is qualified because not everyone uses it or is in favour of its use; it is not used routinely at every meeting, but there is an implicit sense of when it is appropriate to use QuestMap and IBIS on a project. It is brittle because it is not clear if, or for how long, the system would continue to be used if Hertel were not the Director of the Division.

QuestMap has many features, and can be described in several different ways: as groupware, as hypertext, as a Common Information Space, and as an IBIS-based CSAV system. This makes it harder to isolate factors specific to CSAV usage, because users may, for example, be simply tolerating the IBIS component of the system because of the value of the CIS or hypertext functionality. In any case, the CIS and meeting facilitation applications make very different use of CSAV.

Of the 50 staff in SCE's EAD, approximately 10 are moderate users of QuestMap (Hertel, personal communication). "The other 40 are not against the system, they simply do not see a need," Hertel said. "Not many people are willing to embrace the IBIS process and logic. It's probably like the percentage of people who are willing to go to law school and learn that thinking and analysis process. It's hard work!"

One way to measure success would be to look at statistics of system usage over the years. Who was using the system, how often, for what purpose? Selvin and Sierhuis (1999) report a statistical analysis of their QuestMap database. Unfortunately, it has not been possible as of this writing to gather that data from the SCE QuestMap database, which is now approximately 200 megabytes.

\subsection{The Facilitator Issue}

A principal distinguishing feature of the Dialog Mapping approach to collaborative technology is that it does not require everyone to learn new tools and methods. Instead, Dialog Mapping introduces a new player - the Facilitator - into the process, to act as the knowledge mediator, leaving participants free to focus on sharing their knowledge and completing the analytic task at hand.

One natural concern about Dialog Mapping's facilitation approach is, "Where do the Facilitators come from?" Typically, it starts with one enthusiast whose work impresses colleagues. They may of course then want to learn it themselves through informal practice and/or formal training, but a range of 
other options exist, which are being trialled in a variety of organisations affiliated to the Compendium Institute. ${ }^{15}$

- A contracting organization can be a source of Facilitators on an as-needed basis.

- One can offer Dialog Mapping training to managers and team leaders and let those for whom it is a good fit emerge as natural Facilitators. An issue to emerge from a recent workshop ${ }^{16}$ is precisely how much training one needs to reap immediate benefits, and whether one could even envisage adept secretarial staff doing Dialog Mapping instead of just writing minutes.

- In a large organization one could include Dialog Mapping facilitation as one of the skills of the support staff. The Dialog Mapping Facilitator in this setting is simply another standard support function - just as you would always have IT staff to set up and maintain the IT and multimedia platform in a modern, high-tech "fusion" center, you might also have a cadre of facilitators equipped to facilitate using Dialog Mapping tool suite, integrated with the organisation's existing infrastructure.

\subsection{Synchronous versus Asynchronous CSAV}

As we have seen, SCE uses CSAV in two very different ways:

- Synchronous: Dialog Mapping with QuestMap as a facilitation tool during group meetings

- Asynchronous: QuestMap as an organizational memory and file management tool, by individuals in their offices.

The original intent when the SCE Environmental Affairs group acquired QuestMap was to capture organizational memory in an asynchronous usage mode, and that was the "product" that Corporate Memory Systems Inc. was offering. Over time it became clear that, as beneficial as the organizational memory might be, the practical path to successful knowledge capture was by using the system in meetings, i.e., in synchronous mode. This observation accords well with earlier research suggesting that successful adoption of CSAV technology requires a short term payoff for the participants - it cannot simply be a long term payoff documentation effort (Burgess Yakemovic and Conklin 1990).

Ultimately, the practical success of CSAV will depend on an intimate and dynamic relationship between the synchronous and asynchronous modes of CSAV. CSAV tools provide an elegant way to knit two important aspects of knowledge work - individual work at one's desk and conversations during meetings - into a more seamless whole (Selvin, et al., 2002). For instance, Buckingham Shum, et al. (2002) report on how documents are written in the usual asynchronous mode, brought into a meeting and reviewed and modified there, and then new issues and action items captured in the system for further individual work after the meeting. From a practical standpoint, however, these are very different modes of work - they require different kinds of participation, have different tempos and social dynamics, and make very different demands on the supporting CSAV tools. For example, the skill requirements are very different for these two modes. In a meeting, only the Facilitator need be skilled in Dialog Mapping. In asynchronous work using a CSAV organizational memory, all participants must be skillful in the construction and evolution of argument maps. Integrating the practices and tools remains a major challenge for CSAV and for collaborative technology in general.

SCE's experience with working CSAV into everyday work provides some lessons and examples for this integration. For example, during meetings at SCE it is not uncommon to use QuestMap to locate the notes from an earlier meeting, or to locate a document. This is a case of using the organizational memory aspect of the system simply for retrieval during a meeting - no new nodes are created, no Dialog Mapping is done. Conversely, the groupware features of QuestMap allow a map that is under construction during a meeting to be emailed to a staff member with a question or request. Sometimes the staff member is able to quickly answer the question or request by adding new argumentation nodes directly to the map, causing the response to appear automatically on the screen in the meeting room.

The connection between synchronous and asynchronous work seems to focus on documents: how documents (reports, white papers, diagrams, budgets, requirements, etc.) are referenced and used during meetings, and how meeting notes become documents that evolve into other kinds of documents. For example, Selvin and Buckingham Shum (1999; 2002) report on the "representational morphing" of

15 Compendium Institute $<$ www.CompendiumInstitute.org $>$

16 HypACoM 2002: Facilitating Hypertext-Augmented Collaborative Modeling. ACM Hypertext'02 Workshop, 11-12 June, 2002, Univ. Maryland, MD <cognexus.org/ht02> 
documents into IBIS maps, and then back to other notational formats such as data flow diagrams and requirements documents.

SCE's use of QuestMap as a document management system illustrates this connection between the informal knowledge typically captured in CSAV documents and the more formal knowledge of traditional documents, and also illustrates how useful the hypertext features of QuestMap were adapted to the document-centric nature of asynchronous work.

First, many environmental projects organize their project-related documents in QuestMap. The document file itself is stored in a folder in the corporate file system, usually in a subfolder named for the project. The person who saves the file also makes a link to it in QuestMap, by creating a "Reference node" that links to the file (contains the file's path name in the "File name" field of the node). He or she also creates a descriptive Label for the node, and might also enter a brief description of the document or its connection to the project in the Detail field. Finally, the Reference node is graphically linked to the IBIS node or nodes that it is about (see Figure 3). Although creating the QuestMap link is an added step, many EAD staff accept it as necessary because it captures the context in which the document is relevant to the project, because it allows the document to be more easily located using QuestMap's search engine, and because once linked the document's Reference node can be easily reused (transclusively linked) into other maps.

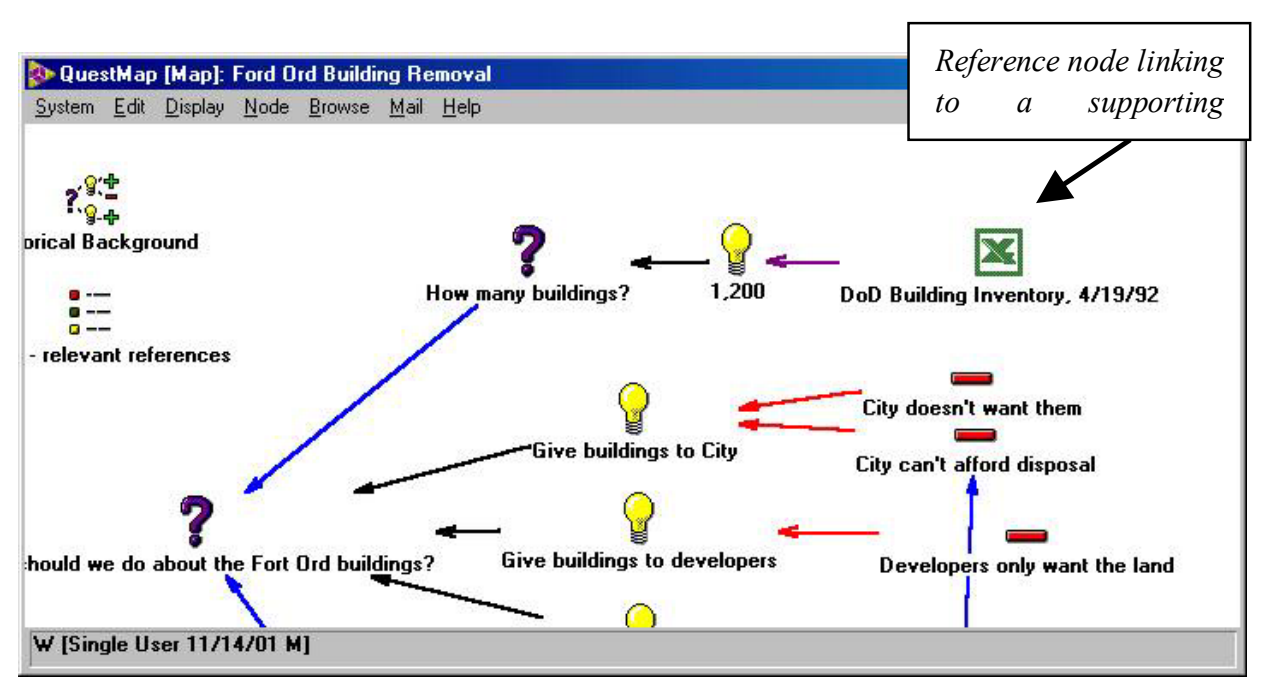

Figure 3: Use of a Reference node to link to an external document (spreadsheet) to back up an answer.

This is not to say that all relevant documents get linked into the appropriate project maps. Indeed, because it is extra work for busy people, relevant documents often do not get linked. Moreover, if a document is moved or renamed, or if new versions are created, the QuestMap links are often not updated. These are not new problems in document management, but they suggest an important set of requirements for extending CSAV tools into the asynchronous mode, to better support the organisational memory function.

Not all of SCE's asynchronous use of QuestMap is as a document management system. Sometimes staff members create argumentation maps asynchronously. One staff member might start a map, for example, seeding it with Questions. Other staff members, invited into the map by an internal QuestMap "email” feature, add new nodes to the map at their convenience (i.e. asynchronously), and reply to the QuestMap email with an indication that they have added to the map. Although this kind of process was the original design focus of QuestMap, in practice most argument maps are created in meetings using Dialog Mapping.

In a second, non-argumentation, use of QuestMap's hypertext features, EAD staff members have evolved a "time-line" map format that they use to organize all of the maps and documents associated with a project. The map consists of a horizontal axis along which major sub-maps are linked in chronological order, and vertical "ribs" extending from any of these major sub-maps with supporting 
sub-maps, notes, or documents (see Figure 4). The individual sub-maps represent CSAV notes of meetings or planning sessions, related maps from other projects, or occasionally time-line maps for subprojects related to the main project ${ }^{17}$.

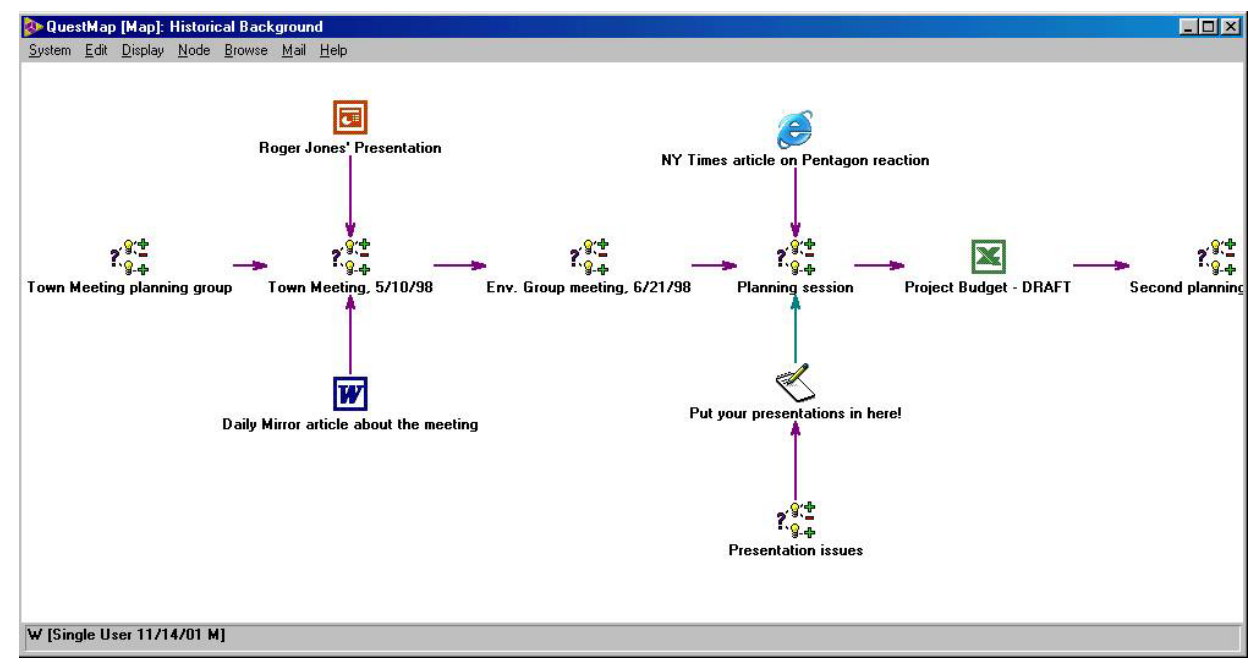

Figure 4: An example of how QuestMap's generic Map container for IBIS discussions has been adapted to other purposes, coupled with Reference nodes to external resources. EAD staff members have evolved a "time-line" map format to organize all of the maps and documents associated with a project, with time progressing left to right.

We have examined SCE's use of the QuestMap CSAV tool as a document management tool. Although this use is not Dialog Mapping, and does not necessarily involve the use of any argumentation, the integration of project documents with the Dialog Mapping argumentation maps is so critical to the overall viability of CSAV at SCE that it bears careful study and further tool support.

\section{$5 \quad$ Introducing Dialog Mapping into a New Organization}

Based on years of Dialog Mapping in business and government contexts, one can distill out a number of factors that have impacted the likelihood of successful introduction. Against these criteria, the 'ideal' pilot experiment for introducing Dialog Mapping to a group would most likely display the following characteristics:

1. The problem chosen should be "medium wicked." The issue or topic that the pilot group is working on should not be a "tame problem," one so simple that observers would say that it could have been solved by using any problem solving approach. Nor should the issue be so wicked that progress on it is virtually impossible due to entrenched positions and political implications. It is not that Dialog Mapping can't be used for such issues; it is just that they don't offer a fair initial evaluation of a new and unfamiliar methodology.

2. There should be a perceived need to increase the level of shared understanding among a group of stakeholders. That is, the ideal issue or topic is one in which there is some history, a wide range of positions and opinions, and some time in which to search for common ground on the topic. This eliminates presentational meetings (one person giving information to a group), and immediate decision meetings (a short time in which a final decision must be taken).

3. The Facilitator should be experienced and well prepared. Much of Dialog Mapping's value rests on the Facilitator; it is important that the person (or persons) filling this role be qualified, experienced, and skillful in all of the aspects of Dialog Mapping. (See Selvin, Chapter 7, for more on Facilitator skills.)

17 The timeline maps are referred to as "Jim Young style maps" at EAD, after the man who created and popularised this format in the group. 
4. The pilot study should have organizational and management support. While there is much to be said for "bottom up" experiments and technology "pull," the ideal situation for a fair evaluation is that the pilot group's management is open to and seeking new ways to do business, and new opportunities for improved collaboration.

5. The experiment should involve a series of meetings. One of the greatest strengths of Dialog Mapping is group memory, the ability to capture and summarize a complex discussion and to carry it forward to future meetings, and for stakeholders who need to be briefed about the meeting. Thus, an ideal evaluation pilot would involve a series of three to six meetings over a period several weeks or months. This would allow the efficiencies of group memory to be demonstrated and evaluated.

6. The participants should care about resolving the issue, and be willing to share information. If the pilot session or sessions are attended by participants who are only there because they were told to be, and who have no intention to collaborate, this is (again) an environment from which little new can be learned.

7. The participants should be open to new approaches and tools.

8. The meeting room should have good quality computer projection, and the seating should be arranged in a " $U$ " that is open to the shared display screen.

9. The technology should not dominate the process. It is the mapping, and the group interaction to craft the map, that is the main value of Dialog Mapping. Indeed, sometimes it is better to start with "manual hypertext" - sticky paper on the wall, whiteboards, sticky notes, chart paper - whatever low-tech display technology is at hand, and then to transition the group to a computer display as the amount of material (and the process of maintaining it) becomes unwieldy.

These criteria are based not just on the SCE experience with QuestMap and Dialog Mapping, but also on experiments using Dialog Mapping in other contexts, including a meeting of analysts in an intelligence organization.

\section{Conclusion and Future Directions}

SCE's Environmental Affairs Division is a unique instance of successful long-term use of a CSAV tool in a business context. While the rarity of such cases points to the relative immaturity of the CSAV and collaborative technology fields, we have reviewed some of the features of SCE's case over the last 10 years that reveal important aspects of successful industrial adoption of CSAV.

Future directions for research include better training, better tools, and deeper understanding of the organizational and social context of CSAV use.

I have spent over ten years developing and refining training materials to teach Dialog Mapping, and I would say there is still much room for improvement and innovation. Being skillful in Dialog Mapping depends on fluency in IBIS, and that fluency, as with any language, comes from practice. Fluency in IBIS is the most challenging skill required, followed by the skill of creating and managing a map in a shared display such that the participants engage with, and own, the map. Naturally, the Facilitator must also know and use the software so well that it is transparent to them during the session. Structured materials and classroom sessions are certainly helpful and necessary, but in the end it seems that the only way to become proficient as a Dialog Mapping Facilitator is to "jump into the deep end and learn to swim," i.e. to set yourself up working on real (and often wicked) problems, and map participants' conversations in a shared display.

One way to make these skills more accessible would be to have a range of training videos from real Dialog Mapping sessions that could be used to illustrate common meeting situations and skillful ways of dealing with them. Another tool might be interactive exercises on the web or on CDROM that presented a meeting situation, allowed the student to create a fragment of a map, and then presented an idealized map along with the reasons for its structure.

If we can find ways to make it easier to learn the skills of Dialog Mapping, it can become more widespread, and that will in turn trigger new innovation and research.

Another front for further research is technology. Ultimately, CSAV is but a part of Doug Engelbart's bigger unfinished revolution to create tools for augmenting the human intellect, both individually and collectively (Buckingham Shum, Chapter 1). Many of the issues raised by the SCE 
experience with QuestMap have to do with integrating argumentation maps with the other aspects of knowledge work: easier and better linking to documents and document elements (figures, paragraphs, etc.), ability to email maps to others (especially colleagues outside of the organization and its firewall), and ability to publish maps and sets of maps on the Internet ${ }^{18}$, and to allow remote participants to contribute to those maps or discussions about them. Selvin, et al. (2002) describes some of the extensions to Dialog Mapping that are emerging as part of the Compendium approach.

Finally, much more research is needed in the social and organizational dimension. Argument maps are a new kind of document. They take a step in direction of being a kind of "living document" that serves as an especially dynamic boundary object among the members of a project team, as well as among subgroups, divisions, and organizations in large projects. As such, argument maps cross the boundary between two familiar metaphors of knowledge work: conversations and publication. During a meeting, a Dialog Map serves as a representation or model of the conversation as it is unfolding. The map is a snapshot of the conversation, but it is also an essential part of the conversation. After the meeting, it is natural to share that captured knowledge more widely, but the metaphor of publication, oriented as it is to more formal and stable kinds of knowledge, requires consideration of such issues as authorship, editing, vetting, validation, scope, and medium. What is the status of a published Dialog Map in a project? It seems to be something new, something more than minutes of the meeting, but less than a traditional report or white paper. What practices and tools are needed to allow social networks to engage with these artifacts in this gray zone between sense-making process and published knowledge representation? How can we make facilitation (in the Dialog Mapping sense) a more natural aspect of the rituals called meetings?

\section{References}

Bannon, L. and Bødker, S. (1997). Constructing Common Information Spaces, Proceedings of the Fifth European Conference on Computer Supported Cooperative Work (ECSCW'97), (Eds.) J. Hughes, et al., Kluwer Academic Publishers, pp. 81-96.

Buckingham Shum, S. and Hammond, N., (1994). Argumentation-Based Design Rationale: What Use at What Cost?, International Journal of Human-Computer Studies, 40, (4), pp. 603-652.

Buckingham Shum, S., Motta, E. and Domnigue, J. (2002). Augmenting Design Deliberation with Compendium: The Case of Collaborative Ontology Design HypACoM 2002: Facilitating Hypertext-Augmented Collaborative Modeling. ACM Hypertext'02 Workshop, 11-12 June, 2002, Univ. Maryland, $\mathrm{MD}<$ cognexus.org/ht02>

Burgess Yakemovic, KC, and Conklin, J. (1990). Report on a Development Project Use of an IssueBased Information System, Proceedings of CSCW'90 (Conference on Computer-Supported Cooperative Work), ACM Press, New York. Also in NCR Journal, NCR, Dayton, Ohio, Vol. 4, No. 1, pp. 37-45, August 1990.

Conklin, J. and Begeman, M. (1989). gIBIS: A Tool for all Reasons, Journal of the American Society for Information Science (JASIS), pp. 200-213, May 1989.

Conklin, J. and Begeman, M. (1989). The Right Tool for the Job, Byte, Vol. 13, No. 10, pp. 255-266, October 1988.

Conklin, J. and Begeman, M. (1988). gIBIS: A Hypertext Tool for Team Design Deliberation, Transactions on Office Information Systems (TOOIS), Vol. 4, No. 6, pp. 303-331, ACM, New York, October 1988. Also in Proceedings of Hypertext'87, ACM Press, New York, 1987.

Kunz, W. \& Rittel, H. (1970). Issues as Elements of Information Systems. Technical Report S-78-2. Insitut für Gundlagen Der Planung I.A., Universität Stuttgart, Kleperstraße 11, 7000 Stuttgart 1, Germany. Also available as: Working Paper 131, Institute of Urban and Regional Development, University of California at Berkeley, 1970.

Selvin, A. (1994) Human Interaction Issues with Group Support Technology in a Software R\&D Organization, Workshop on Human Interaction Issues in Technology Support Environments.

18 The Compendium IBIS mapping tool, a next generation version of QuestMap under development by the Compendium Institute <www.CompendiumInstitute.org> and CoAKTinG Project $<$ www.aktors.org/coakting>, provides powerful web publishing and interoperability with other collaboration tools. 
CSCW'94 Workshop (Conference on Computer-Supported Cooperative Work), ACM Press, New York.

Selvin, A. M. \& Buckingham Shum, S.J. (1999). Repurposing Requirements: Improving Collaborative Sense-Making over the Lifecycle. In Proceedings of International Conference on Product-Focused Software Process Improvement (Profes '99), Oulu, Finland, June 22-24, 1999, 539-559. Full Proceedings: <www.inf.vtt.fi/pdf/symposiums/1999/S195.pdf>

Selvin, A. M. and Buckingham Shum, S. J. (2002). Rapid Knowledge Construction: A Case Study in Corporate Contingency Planning Using Collaborative Hypermedia. Knowledge and Process Management, 9, (2), pp. 119-128. PrePrint available as: <www.kmi.open.ac.uk/tr/abstracts/kmi-tr92.html>

Selvin, A. and Sierhuis, M. (1999). Relative Amounts of Argumentation in Projects Using Differing Structural Conventions. Workshop on Computer-Supported Collaborative Argumentation for Learning Communities, 11-12 Dec., 1999. CSCL'99 Conference, Stanford University, CA $<$ www.kmi.open.ac.uk/sbs/csca/cscl99>

Selvin, A., Buckingham Shum, S., Sierhuis, M., Conklin, J., Zimmermann, B., Palus , C., Drath, W., Horth, D., Domingue, J., Motta, E. and Li, G. (2002). Compendium: Making Meetings into Knowledge Events. Knowledge Technologies 2001, Austin TX March 4-7, 2001 < www.kmi.open.ac.uk/tr/abstracts/kmi-tr-103.html>

Seybold, Patricia (1993). Southern California Edison's Experience with CM/1: Documenting the Decision-Making Process, Paradigm Shift: Guide to the Information Revolution, Vol. 5, No. 1, March 22, 1993. Patricia Seybold Group: Boston, Massachusetts. 